\title{
Ralstonia pickettii
}

National Cancer Institute

\section{Source}

National Cancer Institute. Ralstonia pickettii. NCI Thesaurus. Code C86713.

A species of Gram negative, rod shaped bacteria assigned to the phylum Proteobacteria.

This species is non-fermentative and is not susceptible to most hospital disinfectants. R. pickettii is found in soil and is an emerging nosocomial pathogen that can cause severe infections including bacteremia, meningitis, septic arthritis and osteomyelitis. 